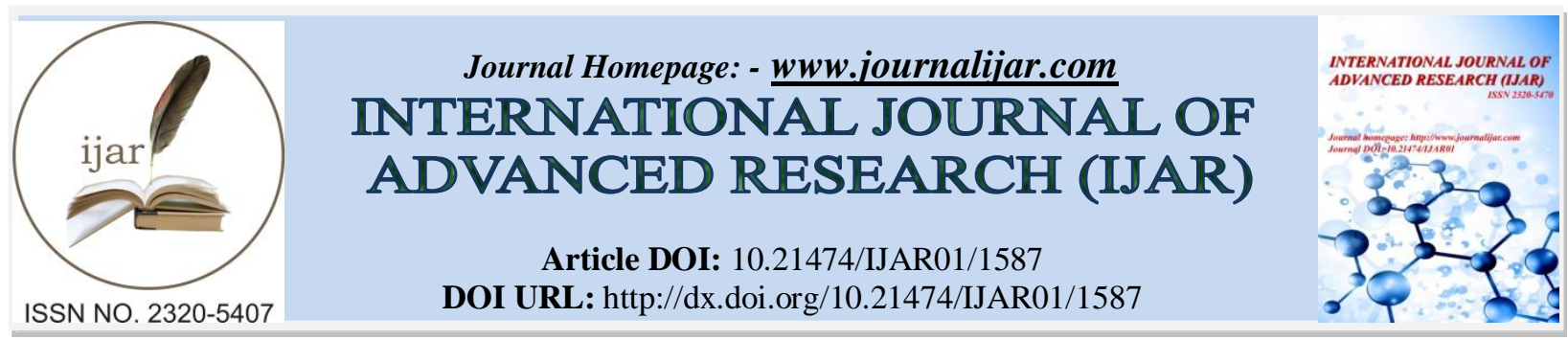

RESEARCH ARTICLE

\title{
EFFECT OF PLYOMETRIC TRAINING PROGRAMME ON SELECTED MOTOR FITNESS COMPONENTS OF SCHOOL STUDENTS.
}

Harbans Lal Godara.

TGT (P\&HE) Kendriya Vidyalaya STPS Suratgarh, Dist Sri Ganganagar Rajasthan 335805.

\section{Manuscript Info}

Manuscript History

Received: 12 July 2016

Final Accepted: 23 August 2016

Published: September 2016

Key words:-

Motor fitness, Plyometric training, agility, balance, speed, explosive strength

\section{Abstract}

The purpose of the study was to investigate the effect of plyometric training programme on selected motor fitness components in adolescents Handball players. The five components of motor fitness are agility, balance, speed, explosive strength and flexibility. Motor fitness is one aspect of the multidimensional construct of physical fitness which is defined as a "set of attributes that people have or achieve that relates to the ability to perform physical activity.

To achieve the purpose, 50 school students aged 14 to 15 years volunteered to take part in this study. The experimental group underwent plyometrics training program three days in a week for six weeks. The subjects were assessed before and after 6 weeks of training. The results of the present study support the use of plyometric training to improve the motor fitness level in the adolescents.

The subjects were divided into two equal groups. The study was formulated as a true random group design, consisting of a pre-test and post-test. The subjects $(\mathrm{n}=50)$ were randomly assigned to two equal groups of twenty five subjects each. The groups were assigned as plyometric training programme group (PTG) and control group (CG) in an equivalent manner. The following are the selected criterion variables; motor fitness variables of explosive strength, muscular endurance, speed and agility. All the subjects were tested immediately prior and after the experimental programme. ' $t$ ' ratio was applied to analyse the significant difference. The .05 level of confidence was fixed as the level of significance to test the ' $t$ ' ratio obtained by the analysis, which was considered as an appropriate. Based on the result of the study it was concluded that, the plyometric training programme produced a significant development on the selected motor fitness components of school students. 


\section{Introduction:-}

Plyometrics are power improvement workouts designed specifically for athletes and advanced exercisers who have a well-conditioned body. Training with this mode of exercise increase muscular strength and improve a specific skill whether it is to jump higher, jump longer, throw farther or hit harder. Systematic plyometric exercises follow a specific patternof muscle contractions. These exercises use movements that develop the ability to generate a large amount of force quickly. The most common exercises from ordinary exercisers for this type are jumping rope, jumping jacks, throwing and catching ball on wall, and boxing with a punching bag. These are usually practiced under supervision by fitness experts or by athletes' coaches. There are techniques and rules to follow when training with plyometrics especially if you are training for a specific sport. Plyometrics began being used in the late 1960s by Russian track and field athletes. The actual term Plyometrics was first coined in 1975 by Fred Wilt, one of the America's more forward-thinking track and field coaches. The seemingly exotic exercises were thought to be responsible for the rapid competitiveness and growing superiority of Eastern Europeans in track and field events. Until the 1980s, Plyometrics were strictly used by the track and field athlete. After this period of time, other sports began to apply Plyometrics to their training as well. Plyometrics is defined as exercises that enable a muscle to reach maximum strength in as short as possible. The speed-strength ability is known as power. For an exercise to be truly plyometric, it must be a movement proceeded by an eccentric concentration. These results not only stimulating the proprioceptors sensitive to rapid stretch, but also in loading the serial elastic components (the tendons and crossbridges between fibers) with a tension force form which they can rebound. A reasonable amount of flexibility is important when beginning the plyometric training program. Plyometric training should be a continuum. It starts simply, and as the athlete matures and develops physically, the system becomes more complex and skill-Specific. It can truly be a piece of the elite performance puzzle. Plyometric training is a specific work force the enhancement of explosive strength. It improves the relationship between maximum strength and explosive strength. Plyometric training utilizes elastic energy and myotic reflex in the development of power. The muscle will resist over stretching and the kinetic energy development in the amortization phase will be utilized to cause a powerful contraction to prevent overstretching of the affected muscle. Thus the momentum generated by the athlete acts as the overload to stretch eccentrically the muscle before concentric concentration, a greater amount of elastic energy is stored in the muscle. This elastic energy is the re-used in the following concentric concentration by making that concentration stronger. The key is the time it takes for the muscle to switch from the lengthening phase to the shortening phase. This leads to the fundamental plyometric principle: the rate, not the magnitude of the strength is what determines the use of elastic energy and the transfer of chemical energy to mechanical work. The improvement in skeletal muscle performance that occurs with initial pre-stretching can be identified as the combined effects of both the storage of elastic energy and the myotic reflex activation of the muscle.

\section{Methodology:-}

The study was designed to assess the effects of a complex training program on the power development of adolescent male Handball players.50 national level handball players aged 14 to 15 years old were selected for the study. The subjects belong to the Kendriya Vidyalaya STPS Suratgarh, Sri Ganganagar, Rajasthan. The subjects were healthy. Both the participants and their parent were informed about the nature of the study. The total players were randomly divided into two groups. Experimental group $(n=25)$ and control group $(n=25)$. The groups were assigned as plyometric training group (PTG) and control group (CG) in an equivalent manner. The experimental group underwent plyometric training programme for a period of six weeks with a prescribed schedule. The explosive strength, muscular endurance, speed and agility (vertical jump, sit ups, 50 meters run and shuttle run tests were used respectively) were selected as criterion variables.

The experimental group performed plyometrics exercises, three days per week for 6 weeks. Before each training session, all the subjects performed 10 minutes of low to moderate exercise and stretching for warming up and researcher discussed and demonstrates proper plyometric exercises. Subjects were assessed before and after a six weeks training program for upper and lower body explosive strength according to the Two Hand Medicine Ball Put and Vertical Jump (Glencross, 1960 ) test. Both the tests were performed three times and the highest values were taken in meter (for M.B.P.) and $\mathrm{cm}$ (for V.J.) respectively for statistical analysis. The detail training protocols described in table 1 and table 2. 
Training protocols:-

Plyometric Training Protocol for 1-3 weeks

TABLE: - 1

\begin{tabular}{|l|l|l|l|}
\hline Exercises & Sets & Rept. & Weigh \\
\hline Bench Press & 3 & 8 & $55 \%$ of 1RM \\
\hline Leg Press & 3 & 8 & $55 \%$ of 1RM \\
\hline Front Press & 3 & 8 & $55 \%$ of 1RM \\
\hline Hamstring Curl & 3 & 8 & $55 \%$ of 1RM \\
\hline Tuck Jump & 3 & 10 & Own body weigh \\
\hline Medicine Ball Over Head Throw & 3 & 10 & $3 \mathrm{Kg}$ \\
\hline Hurdle Jump & 3 & 10 & Own body weight \\
\hline Medicine Ball Seated Throw & 3 & 10 & $3 \mathrm{Kg}$. \\
\hline
\end{tabular}

Plyometric Training Protocol for 4-6 weeks

TABLE: 2

\begin{tabular}{|l|l|l|l|}
\hline Exercises & Sets & Rept. & Weigh \\
\hline Bench Press & 3 & 10 & $55 \%$ of 1RM \\
\hline Leg Press & 3 & 10 & $55 \%$ of 1RM \\
\hline Front Press & 3 & 10 & $55 \%$ of 1RM \\
\hline Hamstring Curl & 3 & 10 & $55 \%$ of 1RM \\
\hline Tuck Jump & 3 & 10 & Own body weigh \\
\hline Medicine Ball Over Head Throw & 3 & 15 & $3 \mathrm{Kg}$ \\
\hline Hurdle Jump & 3 & 15 & Own body weight \\
\hline Medicine Ball Seated Throw & 3 & 15 & $3 \mathrm{Kg}$. \\
\hline
\end{tabular}

The training program consisted of 8 exercises in which 4 were resistance training exercises and 4 were plyometric exercises. The resistance exercises were Bench Press, Leg Press, Front Press, and Hamstring Curl. The plyometric exercises were Tuck Jump, Medicine Ball Over Head Throw, Hurdle Jump, and Medicine Ball Seated Throw.

\section{Analysis of data:-}

For analyzing the data' $t$ ' test was used to find out the significant improvement between the pre-test and post-test of experimental group and control group. The .05 level of confidence was fixed as the level of significance to test the ' $t$ ' ratio obtained by the analysis, which was considered as an appropriate.

Table-1 SUMMARY OF ' $t$ ’ RATIO ON SELECTED VARIABLES OF CONTROL GROUP

\begin{tabular}{|c|c|c|c|c|c|c|}
\hline S.No. & Variables & Pre-Test mean $\pm \sigma$ & Post-test mean $\pm \sigma$ & $\begin{array}{l}\text { Mean } \\
\text { difference }\end{array}$ & $\sigma \mathrm{DM}$ & $\begin{array}{l}\text { ' } \mathrm{t} \text { ' } \\
\text { Ratio }\end{array}$ \\
\hline 01 & Explosive strength & $52.13 \pm 5.48$ & $52.06 \pm 5.67$ & 0.07 & 0.81 & 0.51 \\
\hline 02 & Muscular Endurance & $41.4 \pm 3.12$ & $41.46 \pm 2.33$ & 0.06 & 0.34 & 0.23 \\
\hline 03 & Speed & $6.41 \pm 0.45$ & $6.38 \pm 0.46$ & 0.03 & 0.02 & 1.63 \\
\hline 04 & Agility & $16.32 \pm 0.52$ & $16.49 \pm 0.78$ & 0.07 & 0.15 & 1.25 \\
\hline 05 & flexibility & $20.13 \pm 3.12$ & $20.03 \pm 2.15$ & 0.10 & 0.21 & 1.30 \\
\hline
\end{tabular}

An examination of table-1 indicates that the obtained ' $t$ ' ratios were $0.51,0.23,1.63,1.25$ and 1.30 , for explosive strength, muscular endurance, speed, agility and flexibility respectively. The obtained ' $t$ ' ratios on all the selected variables were found to be lesser than the table value of 2.14 at 0.05 level of significance for 1,14 degrees of freedom. So it was found to be insignificant. 
Table-2 SUMMARY OF ' $t$ ' RATIO ON SELECTED VARIABLES OF PLYOMETRIC TRAINING GROUP

\begin{tabular}{|l|l|l|l|l|l|l|}
\hline S. No. & Variables & Pre-Test mean $\pm \sigma$ & Post-test mean $\pm \sigma$ & $\begin{array}{l}\text { Mean } \\
\text { difference }\end{array}$ & $\sigma$ DM & 't' Ratio \\
\hline 01 & Explosive strength & $52.51 \pm 4.17$ & $60.53 \pm 5.08$ & 8.2 & 0.75 & $12.16^{*}$ \\
\hline 02 & Muscular Endurance & $39.26 \pm 4.58$ & $43.8 \pm 3.89$ & 4.54 & 0.63 & $7.23^{*}$ \\
\hline 03 & Speed & $7.22 \pm 0.52$ & $6.34 \pm 0.28$ & 0.88 & 0.11 & $7.74^{*}$ \\
\hline 04 & Agility & $16.51 \pm 0.86$ & $16.40 \pm 0.86$ & 0.11 & 0.02 & $4.48^{*}$ \\
\hline 05 & Flexibility & $20.18 \pm 3.14$ & $22.03 \pm 2.22$ & 1.85 & 0.11 & 5.32 \\
\hline
\end{tabular}

An examination of table-2 indicates that the obtained ' $t$ ' ratios were 12.16, 7.23, 7.74,4.48 and 5.32 for explosive strength, muscular endurance, speed, agility and flexibility respectively. The obtained 't' ratios on all the selected variables were found to be greater than the required table value of 2.14 at 0.05 level of significance for 1,14 degrees of freedom. So it was found to be significant. Hence the hypothesis was accepted. Result of the study revealed that there was a significant improvement on the explosive strength, muscular endurance, and speed due to the varied modalities of resistance training programme

\section{Discussion of finding:-}

From the result of the study due to the training programme experimental group increased in the explosive strength $15.44 \%$, muscular endurance $12.46 \%$, speed $11.13 \%$ agility $1.27 \%$ and flexibility $2.15 \%$ at the end of the treatment. From the findings of the study it was concluded that there was a significant difference in explosive strength, muscular endurance, speed, agility and flexibility between experimental group and control group due to the training. The finding of the study is in par with some of the literatures, Abbas (2009) was concluded that Plyometrics exercises with depth jumping and rebound jumping characteristics are best used in developing muscle strength of the lower extremities. De Villarreal et al. (2009) were found that the plyometric training improves vertical jump height (VJH). Thomas et al. (2009) was concludes that both DJ and CMJ plyometrics are worthwhile training activities for improving power and agility in youth soccer players. These strength level improvements are usually essential in various sports and games or daily routine task. Correctly designed and supervised this type of training program carries no extra overload on adolescents. This is an important part of conditioning that contributes to improved performance of young boys in various sports and games.

\section{References:-}

1. Adams K, O'Shea Jp, O'Shea Kl, Climstein M. The effect of six weeks of squat, plyometric and squatplyometric training on power production. Journal of Applied Sport Science Research. 1992; 6(1):36-41.

2. Ademola Olasupo Abass (2009) Comparative Effect of Three Modes of Plyometric Training on Leg Muscle Strength of University Male Students. European Journal of Scientific Research. Vol.31 No.4, pp.577-582.

3. American Academy of Pediatrics'. (2 001) Strength training by children and adolescent. Pediatrics 107:147 01472

4. De Villarreal, E.S., Kellis, E., Kraemer, W.J. and Izquierdo, M. (2009). Determining variables of plyometric training for improving vertical jump height performance: a meta-analysis. J Strength Cond Res. 23(2):495-506.

5. De Villarreal, ES, Kellis, E, Kraemer, WJ, and Izquierdo, M. Determining variables of plyometric training for improving vertical jump height performance: A meta-analysis. J Strength Cond Res 23: 495-506, 2009.

6. Docherty, D, Robbins, D, and Hodgson, M. (2004) complex training revisited: a review of its current status as a viable training approach. Strength cond J26:52-57.

7. Donald K. Mathews, Measurement in Physical Education - (Philadelphia: W.B saunders Company, 1958)

8. Ebben, WP and Watts, PB.(1998) A review of combined weight training and plyometric training modes: complex training. Strength con 20:18-2 7.

9. Effects of strength training and detraining on children. Journal of strength and conditioning Research. 10(2): 109 - 114.

10. Faigenbaum Ad, Mcfarland Je, Keiper Fb, Tevlin W, Ratamess Na, Kang J, Hoffman Jr. Effects of a shortterm plyometric and resistance training program on fitness performance in boys age 12 to 15 years. Journal of Sports Science and Medicine. 2007; 6:519-525.

11. Faigenbaum, A., Westcott, W., Micheli, L., Outerbridge, A., Long, C., LaRosa Lo ud, Zaichkowsky 1996. The

12. Fleck, SJ and Kraemer, WJ.(2004) Designing Resistance Training Program. $3^{\text {rd }}$ edition Champaign, Human 
Kinetics.

13. Glencross, D.J. (1960), the measurement of muscular power. A test of leg power and a modification for general use.

14. Goran Markovic; Does plyometric training improve vertical jump height? A meta-analytical review; British Journal of Sports Medicine 2007;41, 349-355.

15. Harrison H. Clarke, "Application of Measurement to Health and Physical Education" (5 $5^{\text {th }}$ Edition) Englewood cliffs: N.J.PrenticeInc, 1976.

16. Kravitz, L. (1996). "The fitness professional's complete guide to circuits and intervals". IDEAToday, 14(1), 3243.

17. Thomas, K., French, D. and Hayes, P.R. (2009). The effect of two plyometric training techniques on muscular power and agility in youth soccer players. J Strength Cond Res. 23(1):332-5. 